\title{
Flexible Fiber Nanogenerator with 209 V Output Voltage Directly Powers a Light-Emitting Diode
}

Long Gu, ${ }^{\dagger, \perp}$ Nuanyang Cui, ${ }^{\dagger, \perp}$ Li Cheng, ${ }^{\dagger}$ Qi Xu, ${ }^{\dagger}$ Suo Bai, ${ }^{\dagger}$ Miaomiao Yuan, ${ }^{\dagger}$ Weiwei Wu, ${ }^{\dagger}$ Jinmei Liu, $^{\dagger}$ Yong Zhao, ${ }^{\S}$ Fei Ma, ${ }^{\dagger}$ Yong Qin, ${ }^{*}, \dagger$ and Zhong Lin Wang ${ }^{*}, *, \|$

†nstitute of Nanoscience and Nanotechnology, Lanzhou University, Lanzhou 730000, China

${ }^{\ddagger}$ Beijing Institute of Nanoenergy and Nanosystems, Chinese Academy of Sciences, Beijing 100085, China

${ }^{\S}$ Beijing National Laboratory for Molecular Sciences (BNLMS), Institute of Chemistry, Chinese Academy of Sciences, Beijing 100190, China

"School of Materials Science and Engineering, Georgia Institute of Technology, Atlanta, Georgia 30332-0245, United States

Supporting Information

ABSTRACT: On the basis of a vertically aligned ultralong $\mathrm{Pb}\left(\mathrm{Zr}_{0.52} \mathrm{Ti}_{0.48}\right) \mathrm{O}_{3}$ (PZT) nanowire array fabricated using electrospinning nanofibers, we developed a new type of integrated nanogenerator (NG) with ultrahigh output voltage of $209 \mathrm{~V}$ and current density of $23.5 \mu \mathrm{A} / \mathrm{cm}^{2}$, which are 3.6 times and 2.9 times of the previous record values, respectively. The output electricity can be directly used to stimulate the frog's sciatic nerve and to induce a contraction of a frog's gastrocnemius. The NG can instantaneously power a commercial light-emitting

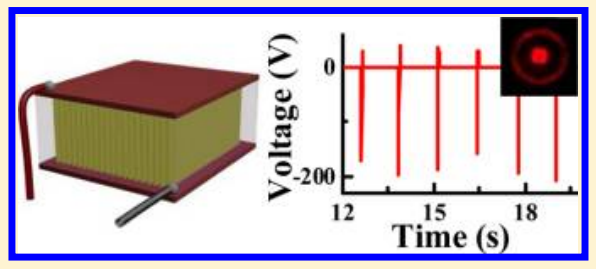
diode (LED) without the energy storage process.

KEYWORDS: Nanogenerator, high output, energy harvesting, PZT nanowires, electrospinning

$\mathrm{H}$ arvesting clean and renewable energy from the environment is an effective method to response the current energy crisis and power wide distributed nano/microdevices. As a novel energy collector, nanogenerator (NG) exhibits a number of features not shared by the traditional generators, that is, the ones based on ocean tide, river falls, and wind, etc. NG fabricated with piezoelectric nanomaterials can convert tiny and irregular environmental mechanical energy to electricity from sources such as air flowing, heart beating, and so on, which are more popular in our living environment compared to the energy source used for traditional generators as mentioned above. Moreover, due to its small size the $\mathrm{NG}$ can be effectively integrated with the nano/microscale functional devices to form a self-powered system, which has potential applications in the internet of things, national security, biomedical, and industry areas. In order to improve its output, many attempts have been made ranging from altering piezoelectric materials, that is, $\mathrm{ZnO},{ }^{2} \mathrm{GaN},{ }^{3} \mathrm{CdS},{ }^{4}$ $\mathrm{PbZr}_{0.52} \mathrm{Ti}_{0.48} \mathrm{O}_{3},{ }^{5,6} \mathrm{BaTiO}_{3}{ }^{7} \mathrm{PVDF}^{8}{ }^{8}$ to different designs, such as lateral, ${ }^{5,9,10}$ radial, $^{11}$ or vertical integrations. ${ }^{2,6} \mathrm{Up}$ to now, various kinds of self-powered functional systems have been realized, such as self-powered $\mathrm{pH}$ sensor, ${ }^{9} \mathrm{UV}$ sensor, ${ }^{9}$ small liquid crystal display, ${ }^{12}$ commercial laser diode, ${ }^{6}$ pressure/ speed sensor, ${ }^{13}$ environmental sensor, ${ }^{14}$ and so on. Among these systems, many of them need an energy storage unit to make them work properly. This energy storage circuit adds much complexity to the self-powered system and hinders its capacity to work in different tough environments. Here, we report a simple approach of fabricating vertically ultralong $\mathrm{Pb}\left(\mathrm{Zr}_{0.52} \mathrm{Ti}_{0.48}\right) \mathrm{O}_{3}(\mathrm{PZT})$ nanowire arrays from electrospinning fibers to make a high output NG. Benefiting from the ultralong length of vertical nanowires, the fabricated NG has a maximum output peak voltage of $209 \mathrm{~V}$, which is much higher than the past record of $58 \mathrm{~V}^{2}$. Also, the NG can output a maximum peak current of $53 \mu \mathrm{A}$ and current density of $23.5 \mu \mathrm{A} / \mathrm{cm}^{2}$, which is 2.9 times of the recent highest value of $8.13 \mu \mathrm{A} / \mathrm{cm}^{2}{ }^{15}$ The output power of our NG can be directly used to stimulate the frog's sciatic nerve and induce a contraction of that frog's gastrocnemius. Moreover, the NG can power a commercial light-emitting diode (LED) instantly without energy storage, which is a considerable progress for the development of selfpowered devices.

Previous studies have shown that high piezoelectric coefficient of the fabricating material and integrated parallel and serial connection designs are two major factors to effectively increase NG's output. So, we use PZT, which possesses the highest piezoelectric coefficient, as our piezoelectric material and adopt a novel design based on ultralong aligned nanowires with length about $420 \mu \mathrm{m}$ in order to get a high output NG. In fact, NG based on the arrays with longer nanowires is equivalent to NGs made up of stacked layers of shorter wires but it can get rid of the superfluous hard substrates and keep nanowire's flexibility. This new method contains the following steps. First, using our reported method ${ }^{16}$ that includes electrospinning the orientated nanofibers and suspending calcination techniques to prepare PZT nanofibers

Received: September 23, 2012

Revised: November 2, 2012 
with an average crystal size of about $16 \mathrm{~nm}$, we obtained a regional orientated film. Second, we cut the orientated PZT nanofiber film and stacked them layer by layer to form a multilayer film as depicted in Figure 1a. Then, the diluted

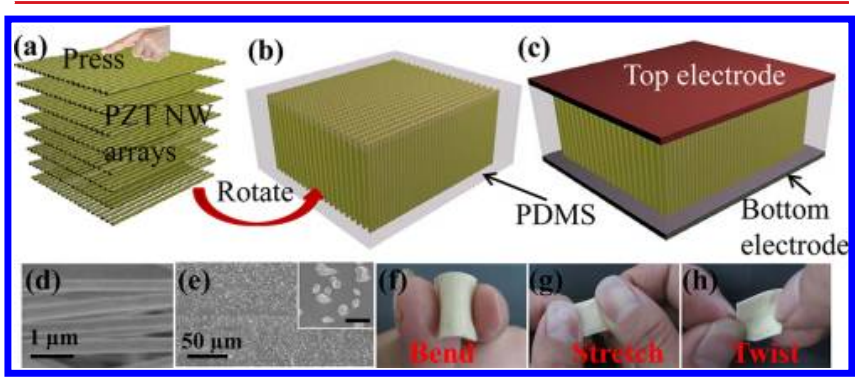

Figure 1. Fabrication process and structure characterization of the NG. $(a-c)$ Experiment setup for fabricating the high-output NG using the regionally orientated electrospinning nanofibers. (d) Fieldemission scanning electron microscope (SEM) image of the regionally oriented electrospinning PZT nanofibers. (e) Top-view SEM images of the fabricated VANA. The inset is a magnified image with scale bar of $2 \mu \mathrm{m}$. The white spots are the exposed tops of PZT nanowires. (fh) Optical photographs of VANA under different deformations that show its flexibility and robustness.

polydimethylsiloxane PDMS $\left(V_{\mathrm{PDMS}} / V_{\text {diluent }}=1: 1\right)$ with low surface tension was filled into the interspace of films and nanofibers with the help of capillary force. Third, we pressed the multilayer film gently to extrude redundant PDMS without damaging its structure. After that, we solidified the PDMS at 80 ${ }^{\circ} \mathrm{C}$ for an hour and then cut off the fringe of the film to remove the nonorientated PZT nanofibers. At last, a laterally aligned PZT multilayer film was obtained. Fourth, we divided the film evenly into a great number of little cuboids, rotated these little cuboids by $90^{\circ}$ simultaneously and then bonded them laterally with PDMS to form a vertically aligned ultralong PZT nanowire arrays (VANAs) as depicted in Figure 1b. Fifth, we fabricated film electrodes on both sides of the VANA to form an NG as depicted in Figure 1c. Figure 1d shows the good orientation of our regional orientated film fabricated in the first step. Figure le is the top-view image of VANA etched by RIE under $\mathrm{O}_{2}$ and $\mathrm{CF}_{4}$ atmosphere for $5 \mathrm{~min}$ from which we can see the arrays in the nanowires of VANA are vertically aligned very well and rather dense. Figure $1 \mathrm{f}-\mathrm{h}$ shows that our synthesized VANA can be bent, stretched, or twisted to a large degree without breaking its structure, which shows its potential application in flexible energy harvesting and self-powered systems.

Before converting mechanical energy into electricity, the NG needs to be poled under a $5 \mathrm{kV} / \mathrm{mm}$ electric field at $130^{\circ} \mathrm{C}$ for $15 \mathrm{~min}$ in order to align the electric domain. Subsequently, the NG can work under the periodic impact given by a heavy object. Its working mechanism is illustrated in Figure 2a,b. As for one period operation of NG, when the heavy object is far away from the NG, no piezoelectric potential is created, as illustrated in Figure 2a. When the moving heavy object is in contact with the immobile NG, an impact pressure is applied on the NG. This force will generate a piezoelectric potential due to the compressive strain of VANA, as shown in Figure $2 b$. This potential will drive the free electrons flow through an external load from the low-potential end to high-potential end and accumulate at the interface of electrode and nanowires to balance the piezoelectric potential. When the object is moved away and the pressure is released, the accumulated electrons will flow back through the load in external circuit. So in each

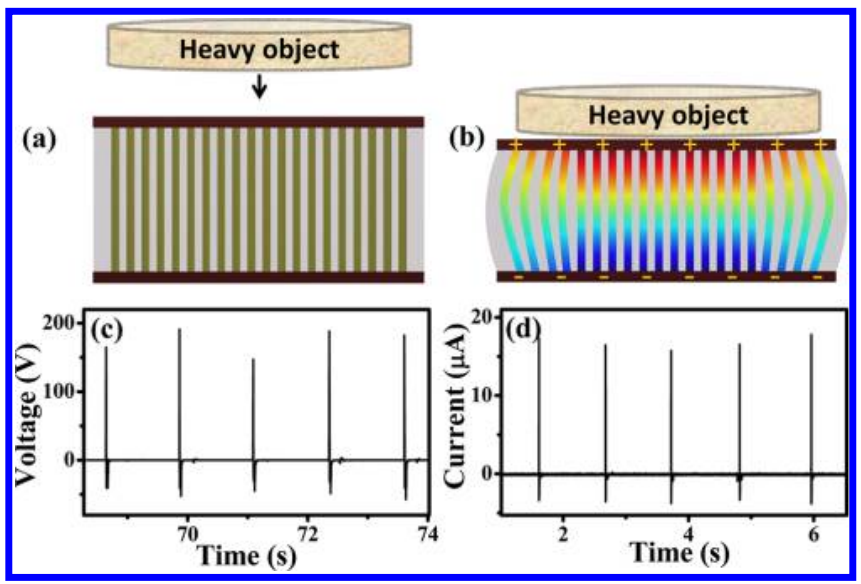

Figure 2. Working principle and performance of the NG. (a) Schematic diagram of NG's structure without deformation. (b) A schematic picture of the piezoelectric potential distribution in a deformed NG when suffer an impact force. The red and blue color regions indicate a positive and negative piezoelectric potential, respectively. (c) Output voltage and (d) output current of the NG under a periodic pressure of $0.53 \mathrm{MPa}$.

period, the positive and negative current and voltage signals are observed. Under a normally periodic impact, the NG with the area of $2.25 \mathrm{~cm}^{2}$ shows a maximum peak output voltage of 198 $\mathrm{V}$ for external load $100 \mathrm{M} \Omega$ (Figure 2c) and a peak output current of $17.8 \mu \mathrm{A}$ for external load $100 \Omega$ (Figure $2 \mathrm{~d}$ ). If larger impact is applied on the NG, it can output a peak voltage of 209 V (Supporting Information Figure S1) and a peak current of $53 \mu \mathrm{A}$ (Supporting Information Figure S2) corresponding to current density of $23.5 \mu \mathrm{A} / \mathrm{cm}^{2}$, which are 3.6 times of the past record output voltage of $58 \mathrm{~V}$ from the integrated nanogenerator ${ }^{2}$ and 2.9 times of the past record output current density of $8.13 \mu \mathrm{A} / \mathrm{cm}^{2}{ }^{15}$

The stimulation of NG to nerve has potential application for the repair of biological neural network. So next we use the instant output electricity of NG to stimulate the frog's sciatic nerve. When the input voltage is beyond the threshold voltage of the resting potential, a contraction of muscle would be induced by opening the valve of the action potential. ${ }^{17}$ For a frog's sciatic nerve, the cells' resting potential ranges from -60 to $-80 \mathrm{mV}$, and a voltage input of at least $50 \mathrm{mV}$ at $1 \mathrm{~Hz}$ is necessary for innervation of the sciatic nerve. ${ }^{18-20}$ So, theoretically our output is large enough to make a contraction of the frog's gastrocnemius. In experiment, an interceptive hind limb of a frog was connected to the NG with the positive and negative terminals of the NG attached to the white corded sciatic nerve (Figure 3a,b). The frog's corded sciatic nerve should be kept wet to maintain its bioactivity throughout the entire experiment process by Ringer's solution for amphibians. The NG was impacted by a single finger as shown in Figure 3a,b. Under the finger's impact, the NG generated electric impulses that were used to stimulate the nerve to cause contraction of the gastrocnemius muscle (Figure 3c,d). Perfect synchronization was observed between the finger's impact and muscle's contraction (video 1 in Supporting Information). This phenomenon is similar to that stimulated by a large NG with area of $9 \mathrm{~cm}^{2}$, which means our much smaller NG with area of $0.95 \mathrm{~cm}^{2}$ can still stimulate the nerve and induce a movement under a weaker impact of tiny finger tap.

Moreover, the output of our NG is high enough to directly power a commercial LED with working voltage of $1.9 \mathrm{~V}$ even 


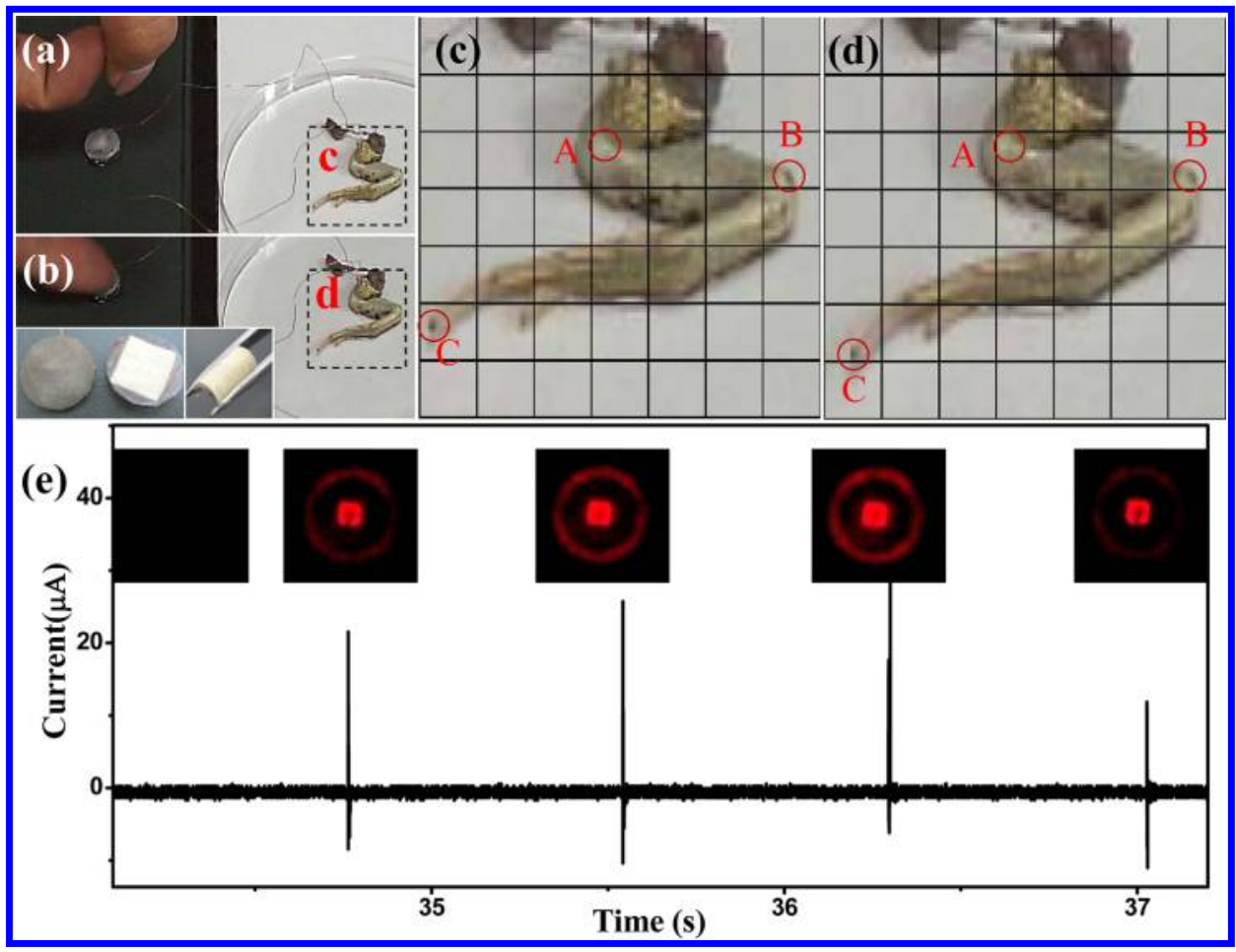

Figure 3. Application of the electric output generated by the NG to stimulate a frog's nerve and drive a commercial light-emitting diode. (a, b) Optical photographs of stimulating a frog's sciatic nerve using NG driven by a single finger's impact. Inset in (b) is the structure of the circular NG, where the white one is the VANA and the gray ones are the electrodes. (c) Optical photograph of the frog's hind leg before the stimulation. (d) Optical photograph of the frog's hind leg stimulated by the NG. Three letters A, B, and C were marked on the three key parts of the frog's hind leg in order to show a relative position angle change when it is stimulated. When the leg was stimulated, the angle between BA and BC changed from 30.75 to $36.13^{\circ}$ (e) Synchronous current signals measured by current preamplifier when the LED is powered by the NG. Insets are the images of the LED's states corresponding to the current signals' position.

when part of output voltage is divided by the inner resistance $100 \Omega$ of current preamplifier. In experiment, we connected a LED, a low noise current preamplifier (Stanford Research System Model SR570), and the NG in series. Under the periodic impacts, transient current and voltage appeared in the external circuit. Powered by this transient electric output, the LED shone red light. Also, the current preamplifier collected a synchronous pulsing signal. Figure $3 \mathrm{e}$ shows four successive pulsing current signals generated by NG, and the LED shone red light for four times in sequence, which are synchronous with the pulsing electric signals as shown in the inset images and video 2 in Supporting Information. The presence of the current preamplifier will consume some energy due to its resistance, so experimentally LED showed a higher luminous intensity without connecting the current preamplifier.

Besides high-output character, our NG can work under the various tiny impacts with different amplitudes and frequencies. As for the situations with different amplitudes, we use the collision induced by a free-falling object with a weight of 193.4 $\mathrm{g}$ at different height ranging from 5 to $13 \mathrm{~mm}$, as shown in Figure $4 \mathrm{a}$, to simulate different degrees of impact. The corresponding voltage and current are shown in Figure $4 b, c$. We used a square root function to fit the relationship between the peaks of the output signal with the free-falling height. We found this function fits the experiment data very well as shown in Figure 4d. This can be roughly interpreted as follows. For the NG, its output signal is proportional to the impact force. The impact force can be estimated using the ratio between the change of momentum and its action time. Since the range of the falling height is not too large, we assume the action time is

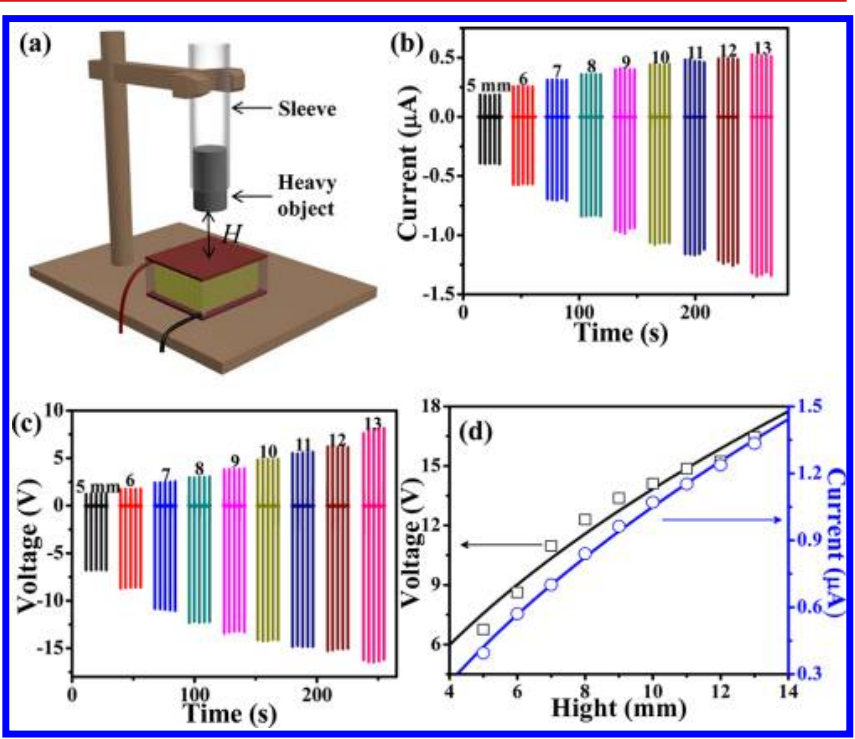

Figure 4. Performance of the NG under different impact forces. (a) Schematic diagram of the drive method. The sleeve is used to make the free-falling object impact the same place in the experiment. $(b, c)$ The current and voltage output of the NG under impact exerted by a free-falling object with different heights, ranging from 5 to $13 \mathrm{~mm}$. (d) Relationship between the peaks of the output voltage (output current) and the height of the free-falling. The square and circle points are experimental data, and the curves are the fitting curves by a square root function.

constant. On the basis of this assumption, the force is proportional to the impact momentum. As for the free-falling 
case, the impact momentum is proportional to the square root of the falling height. So, finally the output signal is proportional to the square root of the falling height.

On the other hand, to study the frequency dependence of NG's output, experimentally we used a linear motor to get a more precise control of the frequency. Different frequencies ranging from 0.25 to $2.5 \mathrm{~Hz}$, were obtained by changing the movement of the linear motor. The response of our NG to different impact frequencies is shown in Figure 5a,b. The

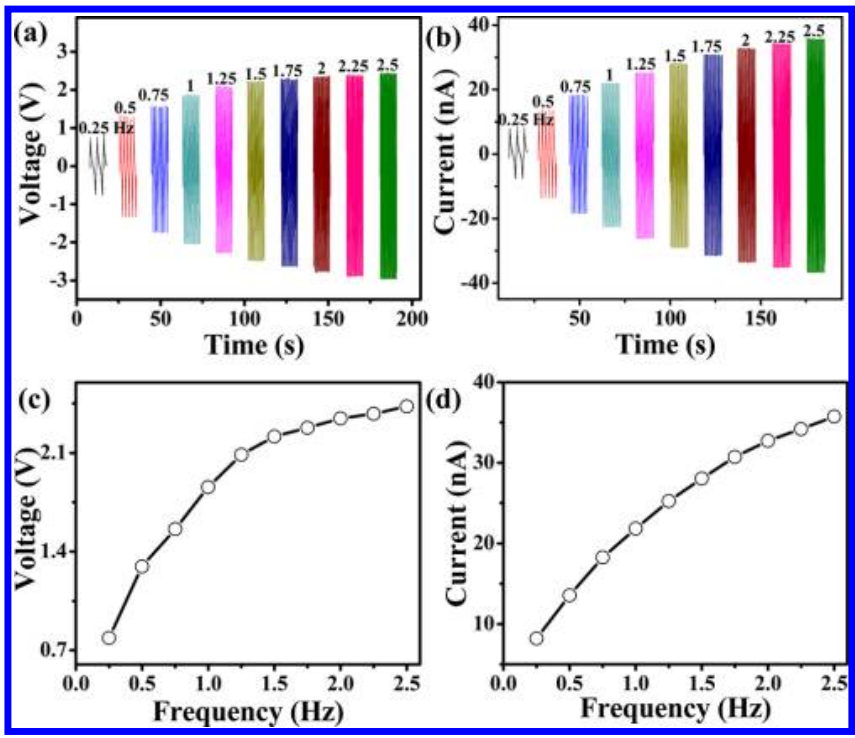

Figure 5. Performance of the NG under different impact frequencies. $(a, b)$ The output voltage and output current of the NG under different frequencies ranging from 0.25 to $2.5 \mathrm{~Hz}$. (c,d) Relationship between the peaks of the output voltage (output current) and the impact frequencies.

relationship between the frequency and the peaks of the output is shown in Figure 5c,d. When we increase the frequency, the electrons in the external circuit have a shorter time to balance the piezoelectric potential, and this will lead to a larger current. As the external voltage is the product of the current and external resistance, the voltage will get bigger correspondingly.

In summary, we have developed a route to fabricate vertically aligned ultralong PZT nanowire arrays and made them into a high output $\mathrm{NG}$, which can easily output a maximum peak voltage of $209 \mathrm{~V}$ and a maximum peak current density of 23.5 $\mu \mathrm{A} / \mathrm{cm}^{2}$. Tiny mechanical movements in wide frequency can be harvested by this kind of NG. The instant output of the NG can be used to stimulate the frog's sciatic nerve to excite a synchronized contraction of frog's gastrocnemius. Moreover, without energy storage the NG can directly power the commercial LED. This kind of flexible high output NG will greatly push forward the application of NGs on self-powered systems, internet of things and so on.

\section{ASSOCIATED CONTENT}

\section{S Supporting Information}

Figure S1 and Figure S2 are output voltage and current signals of NG under a larger impact, respectively. Video 1 and 2 show the induced contraction of frog's gastrocnemius and the shining of the commercial LED powered by our NG, respectively. This material is available free of charge via the Internet at http:// pubs.acs.org.

\section{AUTHOR INFORMATION}

\section{Corresponding Author}

*E-mail: (Y.Q.) qinyong@lzu.edu.cn; (Z.L.W.) zhong.wang@ mse.gatech.edu.

\section{Author Contributions}

${ }^{\perp}$ These authors contributed equally.

Notes

The authors declare no competing financial interest.

\section{ACKNOWLEDGMENTS}

We gratefully acknowledge the financial support from NSFC (No. 50972053), Fok Ying Tung education foundation (131044), Ph.D. Programs Foundation of Ministry of Education of China (No. 20090211110026), the Fundamental Research Funds for the Central Universities (No. lzujbky-2010k01).

\section{REFERENCES}

(1) Wang, Z. L.; Song, J. H. Science 2006, 312, 242-246.

(2) Zhu, G.; Wang, A. C.; Liu, Y.; Zhou, Y.; Wang, Z. L. Nano Lett. 2012, 12, 3086-3090.

(3) Lin, L.; Lai, C. H.; Hu, Y.; Zhang, Y.; Wang, X.; Xu, C.; Snyder, R. L.; Chen, L. J.; Wang, Z. L. Nanotechnology 2011, 22, 475401.

(4) Lin, Y. F.; Song, J.; Ding, Y.; Lu, S. Y.; Wang, Z. L. Appl. Phys. Lett. 2008, 92, 022105.

(5) Chen, X.; Xu, S.; Yao, N.; Shi, Y. Nano Lett. 2010, 10, 21332137.

(6) Xu, S.; Hansen, B. J.; Wang, Z. L. Nat. Commun. 2010, 1, 93.

(7) Park, K. I.; Xu, S.; Liu, Y.; Hwang, G. T.; Kang, S. J. L.; Wang, Z. L.; Lee, K. J. Nano Lett. 2010, 10, 4939-4943.

(8) Cha, S.; Kim, S. M.; Kim, H.; Ku, J.; Sohn, J. I.; Park, Y. J.; Song, B. G.; Jung, M. H.; Lee, E. K.; Choi, B. L.; Park, J. J.; Wang, Z. L.; Kim, J. M.; Kim, K. Nano Lett. 2011, 11, 5142-5147.

(9) Xu, S.; Qin, Y.; Xu, C.; Wei, Y.; Yang, R.; Wang, Z. L. Nat. Nanotechnol. 2010, 5 (5), 366-373.

(10) Zhu, G.; Yang, R.; Wang, S.; Wang, Z. L. Nano Lett. 2010, 10, $3151-3155$.

(11) Qin, Y.; Wang, X.; Wang, Z. L. Nature 2008, 451, 809-813.

(12) Hu, Y.; Zhang, Y.; Xu, C.; Zhu, G.; Wang, Z. L. Nano Lett. 2010, 10, 5025-5031.

(13) Hu, Y.; Xu, C.; Zhang, Y.; Lin, L.; Snyder, R. L.; Wang, Z. L. Adv. Mater. 2011, 23, 4068-4071.

(14) Lee, M.; Bae, J.; Lee, J.; Lee, C. S.; Hong, S.; Wang, Z. L. Energy Environ. Sci. 2011, 4, 3359-3363.

(15) Lee, K. Y.; Kumar, B.; Seo, J. S.; Kim, K. H.; Sohn, J. I.; Cha, S. N.; Choi, D.; Wang, Z. L.; Kim, S. W. Nano Lett. 2012, 12, 19591964.

(16) Wu, W.; Bai, S.; Yuan, M.; Qin, Y.; Wang, Z. L.; Jing, T. ACS Nano 2012, 6, 6231-6235.

(17) Raymond, S. A. J. Physiol. 1979, 290, 273-303.

(18) Frankenhaeuser, B. J. Physiol. 1957, 135, 550-9.

(19) Dodge, F. A.; Frankenhaeuser, B. J. Physiol. 1958, 143, 76-90.

(20) Schalow, G.; Schmidt, H. Pfluegers Arch. 1977, 372, 17-22. 